\title{
Volcanic outgassing and magmatic volatiles
}

\author{
MARIE EDMONDS
}

Earth Sciences Department, University of Cambridge, Downing Street, Cambridge CB2 3EQ, United Kingdom

Exsolved volatiles in magmas have an influence on volcanic and magmatic processes that far outweighs their relatively low abundances. Volatiles modify the rheological and physical properties of magmas during storage and transport; and they play a role in triggering and driving volcanic eruptions, as well as in geochemical cycling, the formation of ore deposits and in the generation of geothermal energy. In this lecture I will review the history of the field of volcanic outgassing and the current state-of-the-art in terms of both our understanding and technological development. Much of the pioneering work in this field was begun or progressed significantly at Kīlauea Volcano, which presented a unique 'laboratory' setting for gas and broader 'volatiles' studies over more than 100 years. 'There is no place on the globe so favorable for systematic study of volcanology ... as in Hawai 'i... where the Earth's primitive processes are at work making new land and adding new gases to the atmosphere" Thomas A. Jaggar, 1916. I will review our progress in understanding magmatic volatile behaviour, based on experimental and petrological studies, and the remaining critical gaps in knowledge. Records of magma degassing, such as melt inclusion suites, have myriad challenges related to understanding post-entrapment processes. Yet, significant progress has been made in unravelling these processes using technological advances in micro-analysis as well as in understanding the origin and petrogenetic history of the crystal hosts. Volcanic gases are the products of magma degassing in the crust during storage and ascent to the surface; I will review the rapid rate of development of this field over the past decade and where the next challenges may lie. Future step changes in progress in understanding magmatic volatiles and their role in Earth processes will come through advances in techniques and capability, but also through intersection of this field with geobiology, tectonics, mantle dynamics and atmospheric chemistry. 